\title{
Liquid Cell TEM Observation of Platinum Based Alloy Nanoparticle Growth
}

\author{
Liyun Zheng ${ }^{1,2}$, Wen-I Liang ${ }^{1}$, Karen Bustillo ${ }^{3}$, Haimei Zheng ${ }^{1,4}$ \\ ${ }^{1 .}$ Materials Sciences Division, Lawrence Berkeley National Laboratory, Berkeley, California, United \\ States \\ 2. College of Materials Science and Engineering, Hebei University of Engineering, Handan, Hebei, \\ China \\ 3. Molecular Foundry, Lawrence Berkeley National Laboratory, Berkeley, California, United States \\ 4. Department of Materials Science and Engineering, University of California, Berkeley, California, \\ United States
}

Platinum based alloy nanostructures have attracted significant interest due to many potential applications, for instance, in catalysis [1,2] fuel cells [3] solar cells [4] and data storage [5]. An understanding and controlling of the growth are essential to achieving the structure and morphology dependent physical and chemical properties. Real time observation of the nanoparticle formation using liquid cell transmission electron microscopy (TEM) is a uniquely important approach.

We prepared the growth solution by dissolving $\mathrm{Pt}(\mathrm{acac})_{2}, \mathrm{M}-(\mathrm{acac})_{2}(\mathrm{M}=\mathrm{Fe}, \mathrm{Ni}, \mathrm{Mn}, \mathrm{Cr}$, etc.) in benzyl ether in the presence of oleylamine and oleic acid. Under electron beam nucleation and growth of Pt-M alloy nanoparticles were achieved.

As shown in Figure 1, Pt-Fe alloy nanoparticles has been obtained a liquid cell [6]. The possible formation mechanisms are highlighted in Figure 1(c). Since platinum ions can be easily reduced to metal under the electron beam due to the high redox potential. Once Pt nanoparticles are nucleated, oleylamine ligands $\left(\mathrm{R}-\mathrm{NH}_{2}\right)$ prefer to bind with Pt surface. As Pt catalyzes the electron transfer between oleylamine and iron ions, $\mathrm{Fe}^{3+}$ ions can be reduced on the platinum nanoparticle surface and they rearrange to form $\mathrm{Fe}_{3} \mathrm{Pt}$. When Pt ions are depleted in the solution, the Pt-Fe alloy nanoparticles cease the growth and iron oxide are observed surrounding the $\mathrm{PtFe}_{3}$ core.

The growth of Pt-Ni nanoparticles shows a two-stage process [7]. As shown in Figure 2, large NiO dendrites are formed at the early stage. They transform into small Pt-Ni nanoparticles subsequently. The resulting Pt-Ni nanoparticles have a narrow size distribution with an average diameter of $4 \mathrm{~nm}$, which is smaller than that obtained by traditional nucleation and growth. The ratio of Pt:Ni is close to $1: 1$. This represents an alternative pathway of obtaining PtNi alloy nanoparticles comparing to the direct nucleation and growth of PtNi nanoparticles [8].

References:

[1] Yu, W., Porosoff, M. D.\& Chen, J. G., Chem. Rev. 112 (2012), p. 5780.

[2] Xia, B. Y. et al, Angew. Chem. 127 (2015), p. 3868.

[3] Mani, P., Srivastava, R., Strasser, P., J. Phys. Chem. C 112 (2008), p. 2770.

[4] Xiao, Y. et al, J. Power Sources 278 (2015), p. 149.

[5] Zeng, H. et al, Appl. Phys. Lett. 85 (2004), p. 792.

[6] Liang, W.-I., et al, J. Am. Chem. Soc. 137 (2015), p. 14850. 
[7] Zheng, L. et al. Manuscript for submission.

[8] The authors acknowledge the funding support from DOE BSE Materials Sciences and Engineering Division. She also acknowledges the facility support of the Molecular Foundry at Lawrence Berkeley

National Laboratory (LBNL).
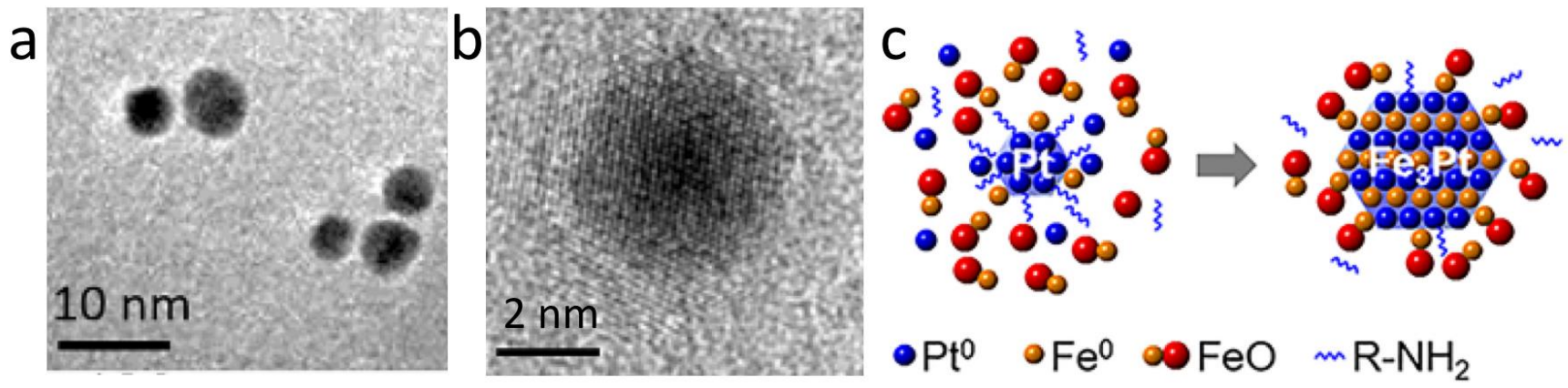

Figure 1. The growth of Pt-Fe alloy nanoparticles observed by liquid cell TEM. (a). A snapshot image of $\mathrm{PtFe}_{3}$ nanoparticles formed in a liquid cell. (b). High resolution TEM image of a $\mathrm{PtFe}_{3}$ nanoparticle in (a). (c). A schematic showing the nucleation and growth of a $\mathrm{PtFe}_{3}$ nanoparticle.
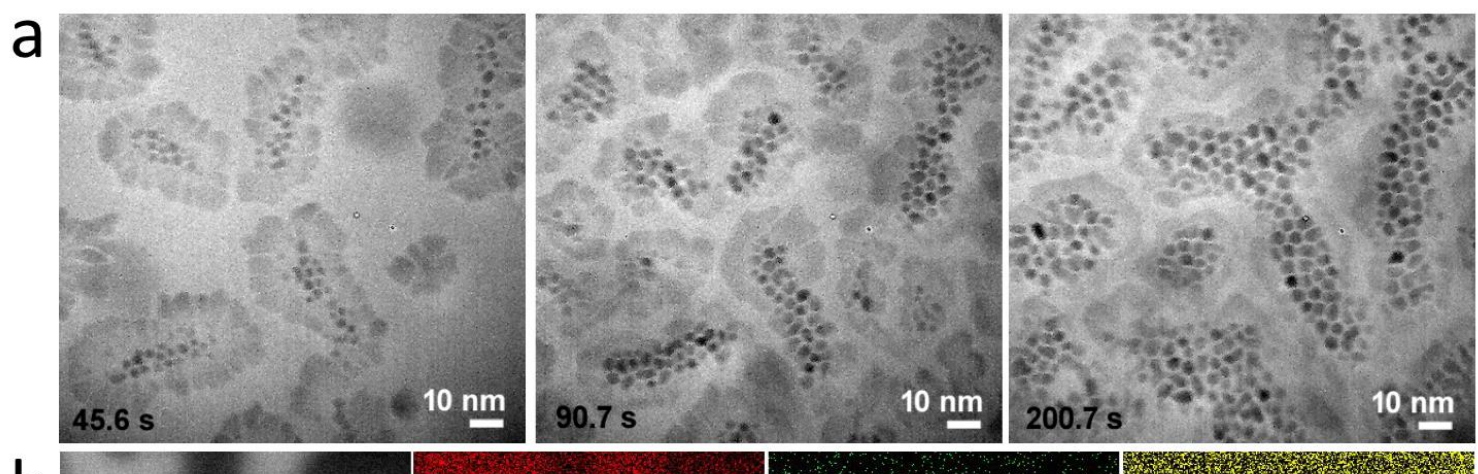

b

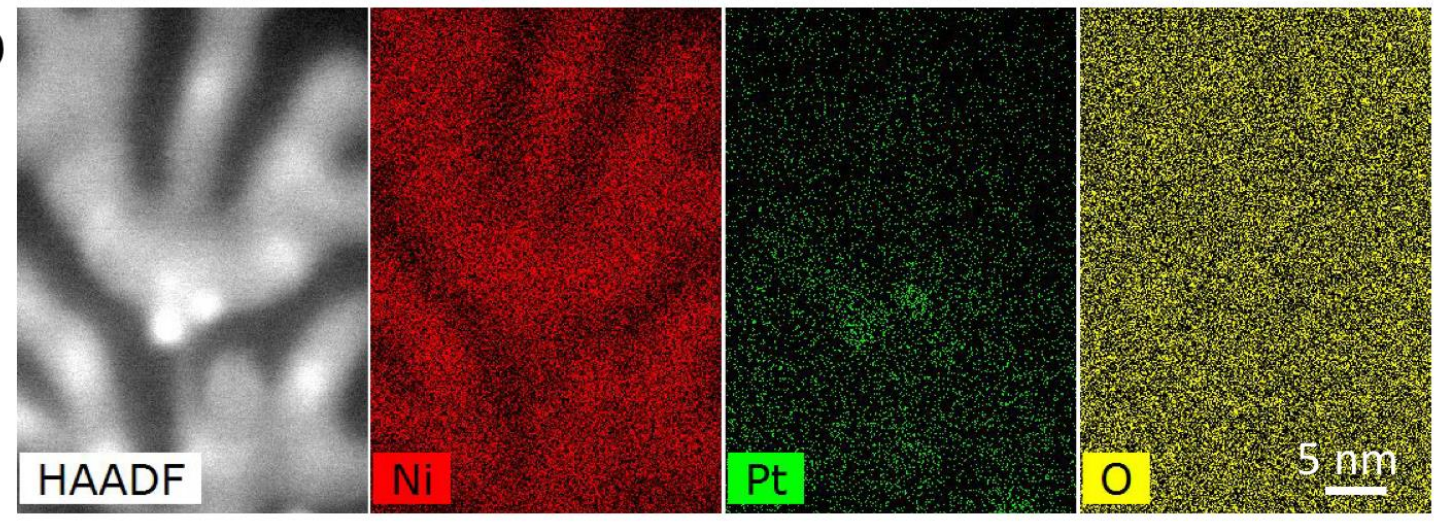

Figure 2. The growth of Pt-Ni alloy nanoparticles observed by liquid cell TEM. (a) Sequential images show the formation of $\mathrm{PtNi}_{3}$ nanoparticles from the initially formed $\mathrm{NiO}$ dendrites. (b) High angle annular dark field (HAADF) image of $\mathrm{NiO}$ dendrites and the nucleation of Pt-Ni alloy nanoparticles. 